\title{
Experimental and Theoretical Investigation into the Polaron Structure of K-Doped Polyfluorene Films
}

Yongzhen Chen, ${ }^{* \dagger}{ }^{\dagger}$ Sarbani Ghosh, ${ }^{\dagger}$ Xianjie Liu, Igor V. Zozoulenko, Mats Fahlman, and Slawomir Braun

Cite This: J. Phys. Chem. C 2021, 125, 937-945

Read Online

\section{ACCESS |}

山lll Metrics \& More

Article Recommendations

Supporting Information

ABSTRACT: The evolution of the electronic structure and optical transition upon n-doping of poly(9,9-dioctylfluorene) (PFO) films is elucidated with photoelectron spectroscopy, optical absorption, density functional theory (DFT), and time-dependent DFT (TD-DFT) calculations. Optical absorption measurements extending into near infrared show two low-energy absorption features at low doping ratios and an additional peak at a higher energy of $\sim 2.2 \mathrm{eV}$ that disappears with increasing doping ratios. A gap state (i.e., polaronic state) close to the Fermi level and a significantly destabilized highest valence band appear in the
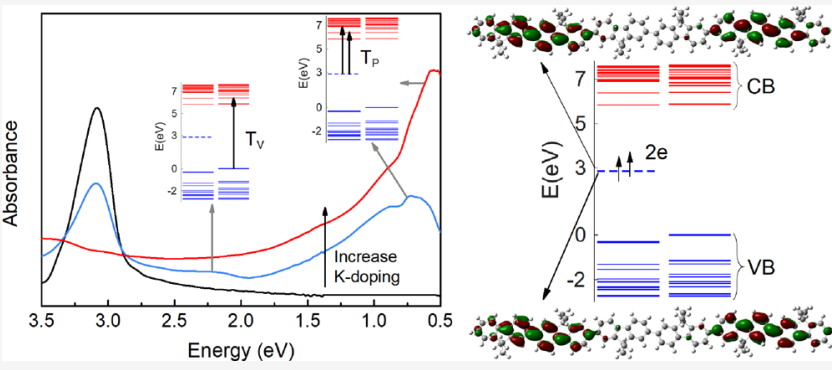
experimentally measured ultraviolet photoelectron spectra. These experimental results are interpreted by the TD-DFT calculations, which show that the lower energy peaks originate from the excitation from polaronic states to the conduction band, while the higher energy peak mainly originates from the destabilized valence band to conduction band transitions and only appears at low doping ratios ( $c_{\text {red }} \leq 50 \%, 0.5$ potassium atom per fluorene monomer). The DFT calculations further indicate that polaron pairs rather than bipolarons are preferentially formed at high doping ratios. Comparing the results of doped glassy and $\beta$-phase films, we find that the ordered segments in the $\beta$-phase film disappear due to the dopant (potassium) insertion, resulting in a similar polaronic structure.

\section{INTRODUCTION}

Polarons, i.e., quasi-particles formed upon electron-phonon coupling, are of the essence in conjugated polymers and are involved in charge transport and exciton dissociation. ${ }^{1-4}$ Polarons are localized charge carriers whose energy levels reside in the forbidden energy gap, and they are affected by the extent of the charge localization and structure reorganization of the polymer ("stiff" vs "soft" conjugated backbone), which is critical to the design of materials for devices. ${ }^{5-8}$ Chemical doping creates polarons through charge transfer from oxidants (p-doping) or reductants (n-doping), ${ }^{9-11}$ and this process is utilized in electrochemical devices such as light-emitting electrochemical cells and organic electrochemical transistors. $^{12-15}$ Chemically doped organic semiconductors are also used as transparent electrode materials, PEDOT:PSS being a widely used example thereof. ${ }^{16,17}$

To better understand their formation mechanism and optical properties, polarons were widely investigated both by experiment and theory in the past decades. ${ }^{1,18-26}$ Occupied polaron states can be directly detected using ultraviolet photoelectron spectroscopy (UPS), ${ }^{26-28}$ and transitions involving polaron states can be probed by UV-vis-NIR optical absorption measurements. ${ }^{20-24}$ The theoretical description of polarons was initially limited by the semi-empirical approaches used, ${ }^{18,29,30}$ but recent work using density functional theory
(DFT) has shed new light on the nature of polaron states in organic semiconductors and the doping-induced optical transitions, largely altering the pre-DFT description of both the electronic structure and optical transitions resulting from polaron formation. ${ }^{31-33}$ The p-doping of organic semiconductors has been extensively studied, as the p-doped organic semiconductors typically are air-stable, which enables easy experimental correlation between UPS data on the (occupied) electronic structure and UV-vis-NIR absorption measurements that provide optical transition energies and indirect data on the unoccupied states. ${ }^{9,33-35}$ Comprehensive studies on the electronic structure and optical properties of $n$ doped organic semiconductors are rare because they require a capability for in situ UPS and vis-NIR spectroscopy to keep the samples in an ultra-high vacuum, water- and oxygen-free environment during sequential doping and measurements.

In our present work, we investigate the polaronic structures of K-doped poly(9,9-dioctylfluorene) (PFO) films by UV-

Received: September 16, 2020

Revised: November 24, 2020

Published: December 22, 2020 

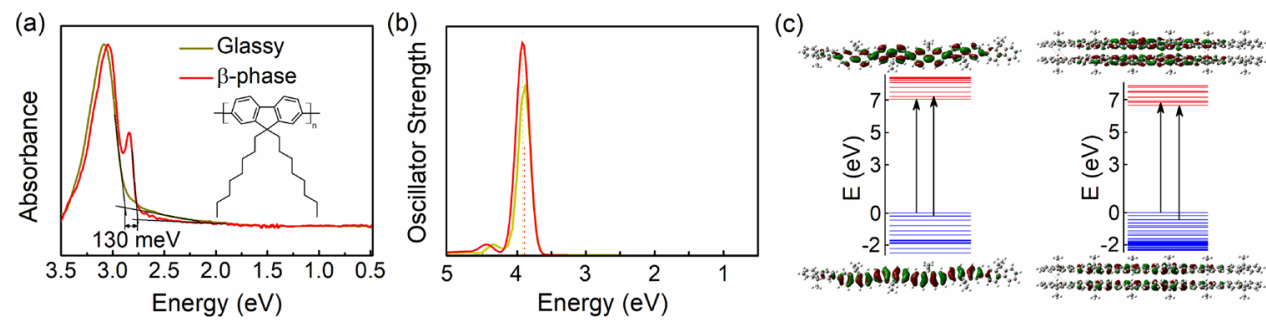

Figure 1. (a) Experimental and (b) theoretical UV-vis-NIR absorption spectra for glassy and $\beta$-phase PFO films. (c) DFT-calculated orbitals and band diagrams of neutral oligofluorene with 6 repeated units $\left(\mathrm{F}_{6}\right)$. (left) amorphous; (right) crystalline. The electron levels in the valence band are depicted as blue lines and in the conduction band are depicted as red lines. The arrows indicate possible optical transition for two phases.

vis-NIR spectroscopy, X-ray photoelectron spectroscopy (XPS), and UPS. Time-dependent DFT-based calculations are used to describe the evolution of absorption peaks with incrementally increasing doping ratios. By comparing the experimental and theoretical results, we provide the assignments of the absorption peaks appearing upon doping. We choose PFO, a common blue polymer used in light-emitting diodes (LEDs), as it has two well-defined conformations, i.e., the disordered glassy phase with an intrachain torsion angle of $135^{\circ}$ and the ordered $\beta$-phase with an intrachain torsion angle of around $180^{\circ} .{ }^{36}$ The latter more planar conformation stems from interchain $\pi-\pi$ stacking during the aggregation process in both films and solutions. ${ }^{37}$ In addition, the two conformations enable us to probe the effects of molecular order, if any, on the doping process and its resulting polaronic structure.

\section{EXPERIMENT}

Materials. PFO used in this study was obtained from Cambridge Display Technology (Cambridge, UK). The solvents such as $o$-xylene, chloroform, and 1,8-diiodooctane (DIO) were all purchased from Sigma-Aldrich. All materials were used as received.

Sample Preparation and Characterization. $\beta$-phasecontaining PFO films were made from $5 \mathrm{mg} / \mathrm{mL} o$-xylene solution mixed with 16 vol \% DIO. Glassy PFO films were made from chloroform solution with a concentration of $5 \mathrm{mg} /$ $\mathrm{mL}$. All samples were spin-coated on pretreated oxidized silicon and glass substrates at $3000 \mathrm{rpm}$ for $60 \mathrm{~s}$. The thickness of all films was about $10 \mathrm{~nm}$.

UPS was performed in a UHV surface analysis system equipped with a Scienta-200 hemispherical analyzer. The excitation source for UPS was a standard He-discharge lamp with $\mathrm{HeI} 21.22 \mathrm{eV}$ and for XPS was a monochromatized $\mathrm{Al} \mathrm{K} \alpha$ radiation with $1486.6 \mathrm{eV}$ energy. The UV-vis-NIR absorption spectra were measured in an optical cavity connected to the UHV system and using the spectrometers Ocean Optics FLAME-T-VIS-NIR-ES (350-1000 nm) and NIR QUEST512-2.5 (900-2500 nm). The full-spectrum curve is a combination of two parts joined at $900 \mathrm{~nm}$. The in situ doping process was carried out by evaporating potassium from a SAES getter source in the UHV preparation chamber. After exposure to the potassium vapor in a controlled step, the sample was transferred to the analysis chamber and optical cavity to finish the measurements. The doping ratio, $c_{\text {red }}$ (i.e., the number of potassium atoms per monomer), is deduced from the XPS result by integrating the peak areas of K $2 p$ and $\mathrm{C} 1 \mathrm{~s}$ and applying appropriate atomic sensitivity factors.

Theoretical Methodology. We calculated electronic structures and optical absorption of fluorene oligomers using the ground-state and the excited-state density functional theory
(DFT), respectively, for the glassy and $\beta$-phase. To simulate the glassy phase, we chose a single PFO chain. Note that experimentally measured absorption spectra are very similar for the glassy and $\beta$-phase. This strongly suggests that the crystallinity of the $\beta$-phase is low. Therefore, to simulate the $\beta$-phase, we choose two $\pi-\pi$ stacked PFO chains as shown in Figure S1. We choose the chain length of the PFO oligomer to be sufficiently long, six monomer units $\left(n=6, \mathrm{~F}_{6}\right.$, corresponding to $78 \mathrm{C}-\mathrm{C}$ bonds in the backbone). For such a long oligomer, it is expected that its optical absorption practically does not change with a further increase in the oligomer length. Note that previous studies demonstrated that as soon as the backbone of conjugated polymers become sufficiently long (48-60 C-C bonds), the optical absorption of the oligomer practically saturates and does not show changes with a further increase in the chain length. ${ }^{38,39}$

All DFT calculations were performed in Gaussian $16^{40}$ without imposing any constraints on initial structures at the $\omega \mathrm{B} 97 \mathrm{X}-\mathrm{D} / 6-31 \mathrm{G}(\mathrm{d})$ level of DFT. ${ }^{41,42}$ The choice of the function used in this study was discussed in our previous study. ${ }^{32}$ We varied the total system charge $(Q)$ on the fluorene hexamer $\mathrm{F}_{6}$ from 0 to 12 , corresponding to the doping ratio $c_{\text {red }}$ from 0 to $200 \%$. All systems were first optimized using groundstate DFT. Following refs 32 and 33, spin-restricted calculations were performed for the charge-neutral systems, i.e., $Q=0$, and for the doped systems with an even number of electrons, i.e., $Q=2,4$, and 6 , for the case of the singlet states. The spin-unrestricted calculations were performed for the doped systems with an odd number of electrons, i.e., $Q=1,3$, and 5, and with an even number of electrons for the case of triplet states. Vis-NIR absorption spectra were simulated by using time-dependent (TD-) DFT at the same level of theory, i.e., $\omega \mathrm{B} 97 \mathrm{XD} / 6-31 \mathrm{G}(\mathrm{d})$, and by applying a similar spin rule.

\section{RESULTS}

Absorption Spectra of Pristine PFO Films. Figure 1a shows the UV-vis-NIR spectra from glassy and $\beta$-phase PFO thin films. The sample preparation process is described in the experimental section in detail. As expected, the glassy film exhibits an unstructured and broadened absorption spectrum, whereas the $\beta$-phase film features a shoulder peak at $2.8 \mathrm{eV}$ with a clearly resolved vibronic structure, which is attributed to a planarized chain geometry with an extended $\pi$-conjugation. As a result, the $\beta$-phase film presents a narrower optical band gap with an energy difference of $130 \mathrm{meV}$ compared to the glassy phase one. In addition, the main band absorption of the $\beta$-phase film redshifts slightly to $3.05 \mathrm{eV}$ due to the appearance

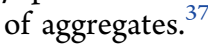

The corresponding absorption spectra obtained by TD-DFT calculations for the fluorene oligomer $F_{6}$ both for the 

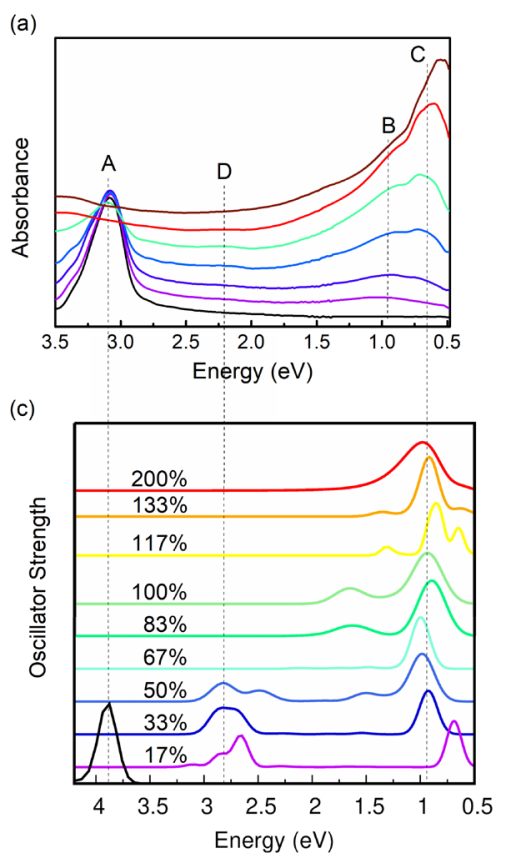

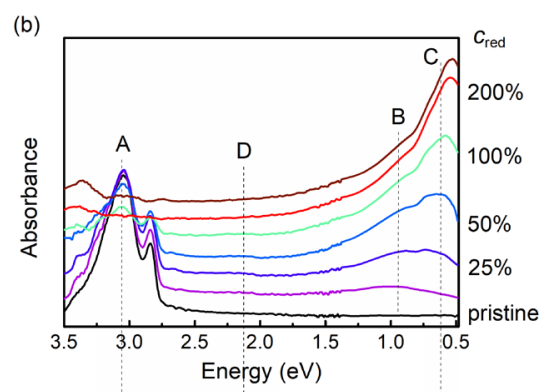

(d)

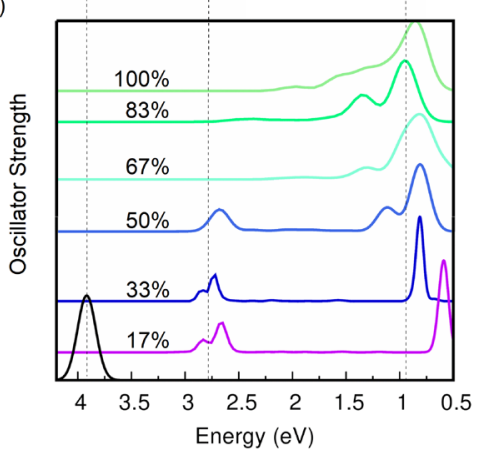

Figure 2. Experimental (top) and theoretical (bottom) UV-vis-NIR absorption spectra of glassy (a,c) and $\beta$-phase (b,d) PFO with incremental doping ratios. The neutral (A) and polaronic (B, C, and D) absorptions are labeled in the figure. The color bar shows the doping ratio derived from XPS (number of $\mathrm{K}$ atoms per fluorene monomer).

amorphous and crystalline conformations are shown in Figure 1b. Figure 1c shows the calculated electronic structure and HOMO (highest-occupied molecular orbital) and LUMO (lowest-unoccupied molecular orbital) orbitals. As expected, the dominant contribution to the absorption peaks of the pristine systems comes from the transitions from the highest occupied to the lowest unoccupied orbitals as indicated by arrows. Note that for the case of two coupled chains (representing $\beta$-phase conformation), the orbitals are extended over two stacked chains because of the interchain coupling.

Absorption Spectra of K-Doped PFO. A series of absorption spectra of PFO doped with increasing stoichiometric $\mathrm{K}$ are presented in Figure 2a,b. The polymer chains are charged by electrons during the doping-induced chemical reduction. The doping ratios are derived from the XPS measurement (see the C 1s spectra in Figure S2). For the glassy film as shown in Figure 2a, only with trace amounts of dopants, the neutral absorption (peak A) weakens, and a new feature $\mathrm{B}$ located at around $1.0 \mathrm{eV}$ appears. It indicates that new states are produced in the band gap by reducing PFO into anions $\mathrm{PFO}^{-}$. With the increasing doping ratio, the absorption of peak $\mathrm{B}$ becomes stronger, and a new peak $\mathrm{C}$ with a lower energy of $\sim 0.7 \mathrm{eV}$ emerges. The latter becomes predominant when the doping ratio reaches one electron per fluorene monomer $\left(c_{\text {red }}=100 \%\right)$.

In the latter stages of the doping, the neutral absorption peak A entirely vanishes, and the absorption peak $B$ is overlapped by peak $\mathrm{C}$. At the same time, the absorption peak $\mathrm{C}$ shifts to the lower energy gradually when the doping ratio is higher than $200 \%$ and ultimately reaches a plateau with an energy of $0.54 \mathrm{eV}$. In addition, another absorption feature $\mathrm{D}$ at around $2.2 \mathrm{eV}$ also appears with a similar evolution as the peak B (see the amplified spectra in Figure S3), which will be analyzed through the UPS results and TD-DFT predictions in the Discussion part. After doping, the sample is exposed to air, and we measure the absorption again, see Figure S4a. It shows a similar profile to the pristine film with only a neutral absorption feature $\mathrm{A}$ in the visible region, indicating that the electronic structure on the polymer chain is largely restored to its pristine state.

The absorption spectra of the K-doped $\beta$-phase film are shown in Figure $2 \mathrm{~b}$. Similar results are obtained as for the glassy sample except for a slight redshift of the peak $\mathrm{C}$ in the $\beta$ phase film (comparison results are shown in Figure S5). It may be caused by the residual polymer chain aggregations remaining even after $\mathrm{K}$ insertion. When the sample is exposed to air after doping (see Figure S4b), only the absorption feature of the pristine glassy phase is reproduced, whereas the $\beta$-phase portion is removed completely. These results indicate that $\beta$-phase chains transform into the glassy phase during $\mathrm{K}$ doping, producing a more disordered film. According to the previous studies, the formation of the $\beta$-phase is induced by van der Waals force between interdigitated side chains during the aggregation of polymer chains. ${ }^{37}$ The presence of $\mathrm{K}$ thus appears to largely break up these aggregated states, likely driving the polymer chains to a more twisted conformation to reduce the spatial steric hindrance.

The optical properties of polarons on PFO chains produced by chemical and electrochemical doping and photo-/electroexcitation have been studied extensively. Due to the different measurement methods and research purposes, the absorption spectra present in these works vary somewhat. When measured in solution, $\mathrm{PFO}^{-\cdot}$ anions produced by chemical reduction or electroexcitation show two clear absorption bands: one at the NIR region $(0.5 \mathrm{eV})$ and the other one at the visible region $(2.07 \mathrm{eV}) .^{43}$ The visible band decreases at high chemical reduction. The fluorene oligomers also show similar results in the same experiment. ${ }^{44}$ PFO films measured in photoinduced absorption spectroscopy show a much weaker signal near 2.0 $\mathrm{eV}$, partly due to the low polaron yield. ${ }^{45}$ A low-energy peak resolved in the $\beta$-phase film is assigned to the ordered chains. Another report based on electrochemical reduction focuses on 
the visible region, showing the evolution of the neutral absorption and the newly generated absorption feature around $2.0 \mathrm{eV}$ as the doping level increases. ${ }^{46}$ The comparison of these spectra enables us to better understand our results. First, both for the glassy and $\beta$-phase films, we can still see the absorption peak $B$ as a shoulder of peak $C$ at high doping ratios, which looks similar to the high doping spectra of PFO chemically reduced in solution. Second, similar to the photoinduced absorptions in films, the peak D in our spectra is much weaker than the peaks B and C, while the chemically doped PFO in solution shows a much stronger peak $\mathrm{D}$. This may be caused by the nature of the polaron in the film. Third, the $\beta$-phase peak at $2.8 \mathrm{eV}$ bleaches together with the glassy phase peak, showing a different evolution process compared to the electrochemical reduction, in which the bleaching of glassy phase absorption starts after the $\beta$-phase has vanished.

UPS Spectra of Pristine and K-Doped PFO. Figure 3 displays the UPS spectra of glassy and $\beta$-phase films with
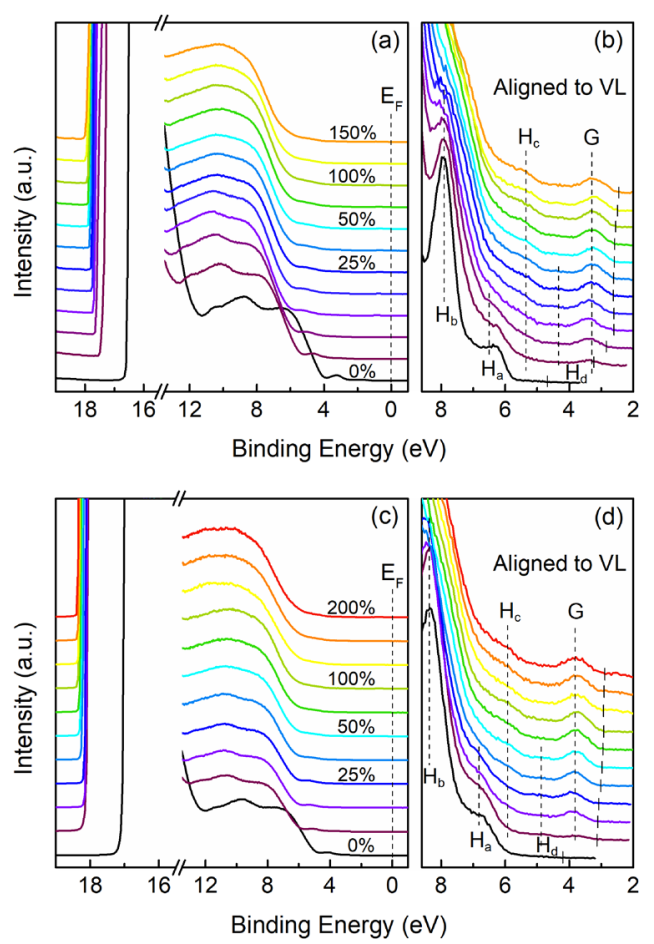

Figure 3. Evolution of UPS spectra with increasing $\mathrm{K}$ doping ratios for glassy (a) and $\beta$-phase (c) films. (b,d) Enlarged spectra in the valence band region after aligning to the vacuum level.

increasing $\mathrm{K}$ doping. The full spectra (Figure 3a,c) are aligned to the Fermi level $\left(E_{\mathrm{F}}=0 \mathrm{eV}\right)$, and the enlarged valence band spectra (Figure $3 \mathrm{~b}, \mathrm{~d})$ are aligned to the vacuum level $\left(E_{\mathrm{vac}}=0\right.$ $\mathrm{eV}$ ). The main doping ratios are marked in the full spectra. Similar to the absorption results, the two conformations show consistent UPS evolution and also agree with those previous reports. ${ }^{4-49}$ Both pristine samples show two peaks at the valence band region, i.e., $\mathrm{H}_{\mathrm{a}}$ and $\mathrm{H}_{\mathrm{b}}$. The ionization potentials (IP) of the glassy and $\beta$-phase PFO are $5.86 \pm 0.10 \mathrm{eV}$. With the first step of $\mathrm{K}$ deposition, the secondary electron cutoff sharply moves to higher binding energy due to the electron transfer to the PFO chains and evolves slowly until the dopants penetrate to the UPS detection depth of $\sim 3-5 \mathrm{~nm}$. Then, it changes very little when increasing the doping ratio further. The $\mathrm{K}$ content increases at the surface and diffuses into deeper layers through a concentration gradient, as determined by angle-resolved XPS (see Figure S6a). With $10 \mathrm{~min}$ annealing, more $\mathrm{K}$ atoms diffuse to the bottom layers and exhibit a weaker intensity in the XPS spectra, as shown in Figure S6b.

Similar to the evolution of the secondary electron cutoff, the peaks $\mathrm{H}_{\mathrm{a}}$ and $\mathrm{H}_{\mathrm{b}}$ of the neutral polymer gradually move away from the Fermi level with a decreased intensity and sharpness, disappearing altogether when the doping ratio reaches $50 \%$. The change is consistent with the peak $\mathrm{A}$ in the absorption spectra described above. At the same time, two new features, i.e., $H_{c}$ and $G$, emerge near the Fermi level and become stronger with increasing doping ratios, see the amplified spectra in Figure $3 \mathrm{~b}, \mathrm{~d}$. The peak $\mathrm{G}$ corresponding to the polaronic states was discussed in previous reports. ${ }^{26-28,50}$ However, $\mathrm{H}_{\mathrm{c}}$ usually seen as the dispersion of the peak $\mathrm{H}_{\mathrm{a}}$ is redefined here. We consider it as a new valence band feature of charged PFO. When the spectra are enlarged further, another destabilized peak $\mathrm{H}_{d}$ can be also distinguished between $\mathrm{H}_{c}$ and $\mathrm{G}$ in the initial stages of doping, as shown in Figure S7. Here, "destabilized" means decreased binding energy, i.e., moves closer to the vacuum level. To our knowledge, we are the first to report this feature, and it is attributed to the miniband lying at the top of the valence band.

Calculated Electronic Structure and Optical Transitions. Figure $2 c, d$ shows the calculated optical transitions of amorphous $\mathrm{F}_{6}$ where chains are charged by electrons from $Q=$ 0 to $12\left(c_{\text {red }}=0-200 \%\right)$. As mentioned above, the calculated spectrum of the pristine $\mathrm{F}_{6}$ shows one absorption peak at 3.9 $\mathrm{eV}$ (peak A), corresponding to the excitations from the highest occupied to the lowest unoccupied orbitals. Once the oligomer becomes charged, the peak A disappears, and two new peaks appear, a weaker peak at higher energy $(E \approx 2.75 \mathrm{eV})$ and a more pronounced one at lower energy $(E \approx 0.75-1 \mathrm{eV})$. The higher energy peak is present in the spectra for $Q=1,2$, and 3 $\left(c_{\text {red }}=17,33\right.$, and $\left.50 \%\right)$. When the doping ratio is increased above $Q=3\left(c_{\text {red }}>50 \%\right)$, this peak disappears, and the spectra are dominated by one or several closely spaced overlapping peaks at lower energies $E \approx 0.75-1 \mathrm{eV}$.

In order to understand the evolution of the absorption spectra, let us consider how the electronic structure changes when the doping ratio is varied. Figure 4 shows the electronic structure for several representative doping ratios $Q=1,2$, and $6\left(c_{\text {red }}=17,33\right.$, and $\left.100 \%\right)$. (Note that $Q=1$ and 2 correspond to the case of lower doping when the spectra exhibit two peaks at $E \approx 2.75 \mathrm{eV}$ and $E \approx 0.75-1 \mathrm{eV}$, whereas $Q=6$ corresponds to the case of higher doping with one peak (or several closely overlapping peaks) at $E \approx 0.75-1 \mathrm{eV}$. Note also that the electronic structure of $\mathrm{F}_{6}$ for the remaining doping ratio is shown in Figure S8.)

Let us start with the case $Q=1\left(c_{\text {red }}=17 \%\right)$ illustrated in the left panel of Figure $4 \mathrm{a}$. When the chain becomes charged, the spin degeneracy is lifted, and a new occupied spin-resolved energy level appears in the gap. This energy level is occupied by an electron, which corresponds to a singly polaronic state of the spin $S=1 / 2$ in the chain. The polaron is localized over $\sim 3$ monomer units, see a corresponding wave function in Figure 4a. Remarkably, at the top of the valence band, there is a miniband separated by a small gap from the deeper lying states. It appears at low doping ratios and is completely consistent with the observed peak $\mathrm{H}_{\mathrm{d}}$ in the UPS spectra. The right panel of Figure $4 \mathrm{a}$ shows the absorption peaks where the dominant transitions between the energy levels corresponding to the largest configuration interaction coefficients as calculated using 

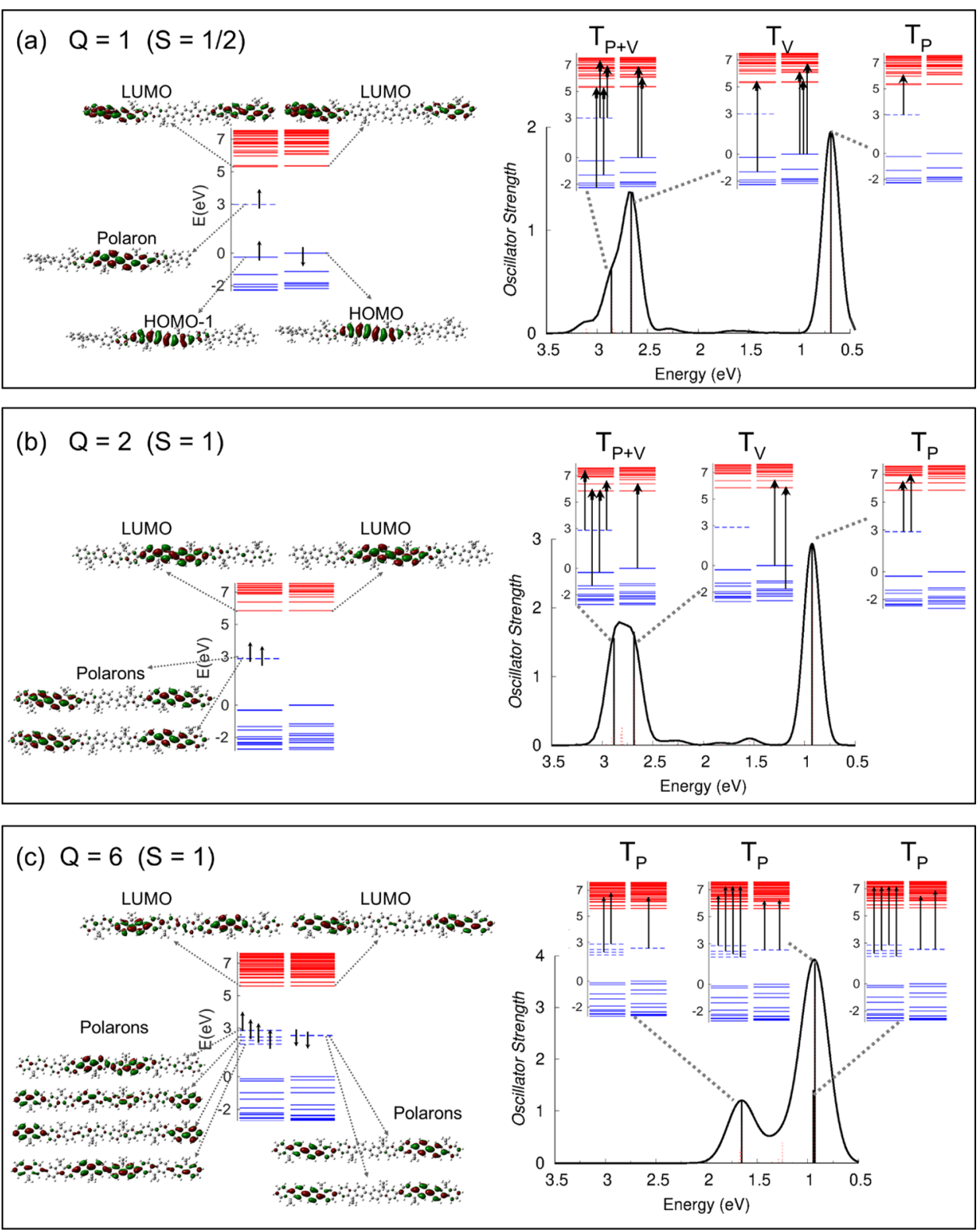

Figure 4. $(\mathrm{a}-\mathrm{c})$ (right panel) DFT-calculated orbitals and band diagrams of amorphous $\mathrm{F}_{6}$ for representative doping ratios $Q=1,2$, and $6\left(c_{\text {red }}=\right.$ 17, 33, and 100\%). (left panel) Calculated absorption spectra and contributions of the corresponding excitations.

TD-DFT are indicated. The higher energy peak $(E \approx 2.75 \mathrm{eV})$ originates from the excitation from the highest occupied level in the top of the valence band to lower lying unoccupied levels in the conduction band. The lower energy peak $(E \approx 0.75 \mathrm{eV})$ is due to the excitation from the polaronic state to lower lying unoccupied levels in the conduction band. These transitions are marked by " $\mathrm{T}_{\mathrm{V}}$ " and " $\mathrm{T}_{\mathrm{P}}$ ", respectively. Note that the higher energy peak also includes a transition from the occupied levels in both valence and polaronic bands to lower lying unoccupied levels in the conduction band. These transitions are marked as " $\mathrm{T}_{\mathrm{P}+\mathrm{V}}$ ". It is noteworthy that the origin of the peaks in the present polymer (i.e., the nature of the transitions " $\mathrm{T}_{\mathrm{V}}$ ", " $\mathrm{T}_{\mathrm{P}}$ ", and " $\mathrm{T}_{\mathrm{P}+\mathrm{V}}$ ") is rather generic and is the same as the one in another $\mathrm{n}$-doped conducting polymer. ${ }^{32}$

Upon further doping, $Q=2\left(c_{\text {red }}=33 \%\right)$, the second electron fills in the orbital with the same spin and slightly higher energy than the first electron, resulting in a triplet state $(S=1)$ corresponding to a formation of a polaron pair, see the left panel of Figure $4 \mathrm{~b}$. Note that the total energy of this state is lower than the bipolaron state (i.e., when two electrons occupy the spin degenerate orbitals of the opposite spins). This can be explained by the Hund's rule that the maximum spin multiplicity is preferential in the ground state of a multielectron system due to the lowest total energy. Note that the formation of polaron pairs instead of bipolarons was also predicted by the DFT calculations for the cases of both pdoped $^{33}$ and n-doped polymers. ${ }^{32}$ The spin multiplicities of the ground state for different doping ratios are summarized in Table $S 1$. For the case $Q=2$ (as well as for the case $Q=3$ ), the origin of the two absorption peaks is the same as for the case $Q$ $=1$, i.e., $\mathrm{T}_{\mathrm{V}}$ transitions for the higher energy peak (with some contribution of $\mathrm{T}_{\mathrm{P}+\mathrm{V}}$ transitions) and $\mathrm{T}_{\mathrm{P}}$ transitions for the lower energy peak, see the right panel of Figure $4 \mathrm{~b}$ and Figure S8.

On further doping, more orbitals appear in the gap, with the number of the orbitals being equal to the number of excess 

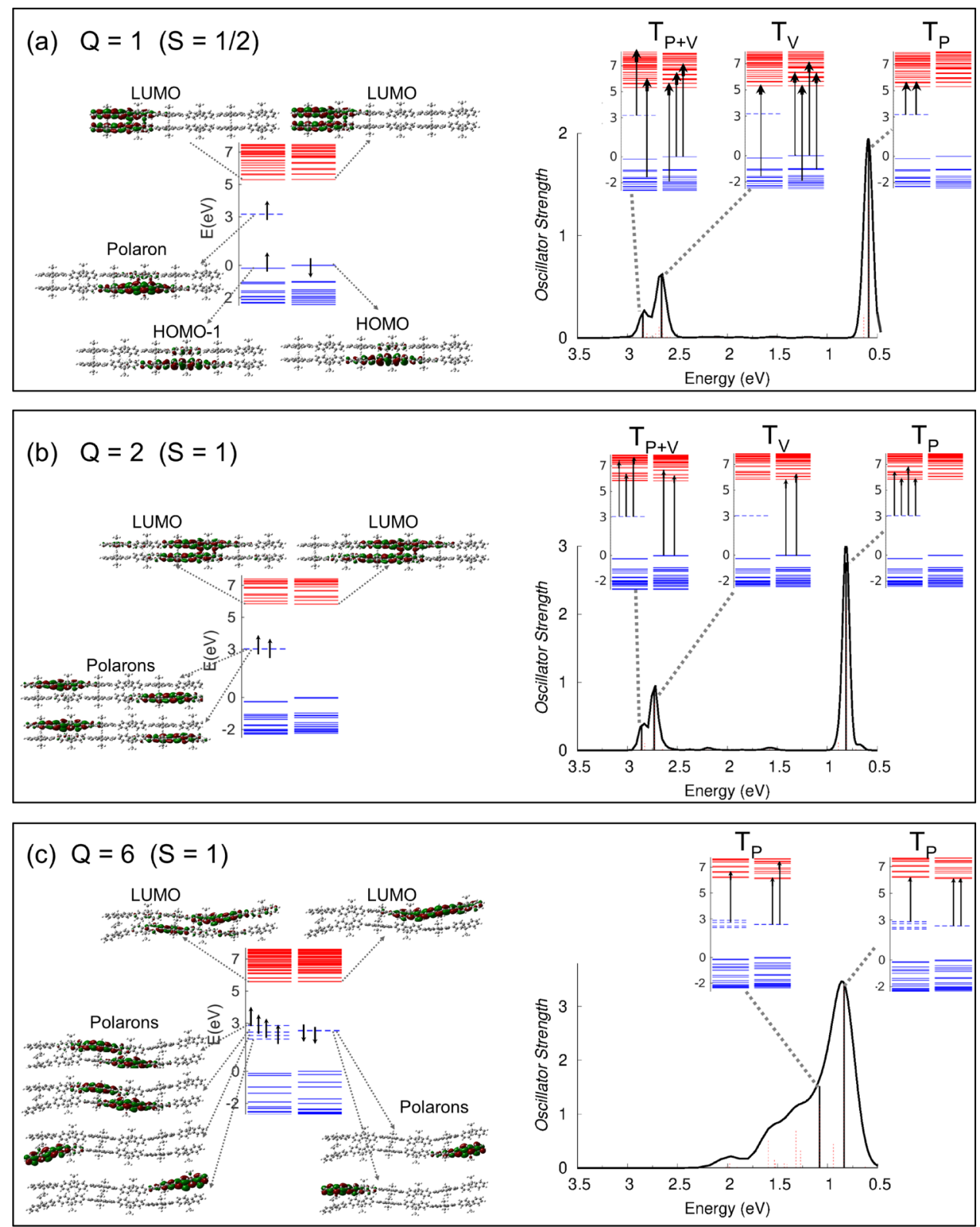

Figure 5. $(\mathrm{a}-\mathrm{c})$ (right panel) DFT-calculated orbitals and band diagrams of the $\beta$-phase for representative doping ratios $Q=1,2$, and $6\left(c_{\text {red }}=17\right.$, 33, and $100 \%$ ). (left panel) Calculated absorption spectra and contributions of the corresponding excitations.

electrons. Figure $4 c$ shows the case of $Q=6$ extra electrons on a chain $\left(c_{\text {red }}=100 \%\right)$. These six electrons occupy six spinresolved orbitals, four of one spin (say, spin-up) and two of the opposite spin (spin-down). This results in the total spin of $S=$ 1 , i.e., the triplet ground state. All peaks in the absorption spectrum correspond to the transitions $T_{P}$, i.e., the excitations from the polaronic states to the lowest unoccupied levels in the conduction band, see the right panel of the figure. Note that this assignment of the peaks in the absorption spectra (i.e., transitions $\left.\mathrm{T}_{\mathrm{P}}\right)$ holds for all other doping ratios $Q>4\left(c_{\text {red }}>\right.$ $67 \%)$.

Let us now turn our attention to the case $\beta$-phase PFO shown in Figure 5 for representative doping ratios $Q=1,2$, and $6\left(c_{\text {red }}=17,33\right.$, and $\left.100 \%\right)$ (see also Figure S9 for all doping ratios $Q=1-6$ ). A comparison of Figures 4 and 5 (see also Figures S8 and S9 for all doping ratios $Q=1-6$ ) shows that the evolution of the electronic structure as a function of the doping ratio and the assignment of the peaks are practically the same as for the glassy (amorphous) film. This is not surprising because the interchain electron interaction is much weaker than the intrachain one, and therefore, the electronic and optical properties of the $\pi-\pi$ stacked chains are still primarily determined by those of individual chains. However, the peak positions of the $\beta$-phase film move slightly toward lower energy in total due to the presence of such interaction.

\section{DISCUSSION}

Let us now analyze the observed absorption spectra of glassy and $\beta$-phase PFO films and their evolution with incremental doping using the results of the DFT calculations. We start with a discussion of the accuracy of the DFT calculations. During the past decades, the DFT approach has de facto become the method of choice for quantum chemistry and computational material science. However, the DFT method has its limitations, and in many cases, the predictions based on this method are not exact but have a rather semi-quantitative character. 
Recently, one of the present authors reported the comparative theoretical and experimental studies of UV to IR absorption of molecularly p-doped polythiophenes. ${ }^{39}$ The structure of the calculated spectra (a number of peaks and their relative positions) was found to be in good agreement with experimental ones; however, the calculated peaks were shifted with respect to the corresponding experimental ones as much as $0.75 \mathrm{eV}$. Moreover, different DFT exchange-energy functionals used in the DFT calculations $(\omega \mathrm{B} 97 \mathrm{XD}$ and B3LYP) resulted in the spectra that showed a similar structure but were also substantially shifted with respect to each other. Therefore, it is expected that the DFT calculations are in a position to provide the semi-quantitative description of the evolution of the spectra but not necessarily the absolute peak positions. This is also the case for the present system where, for example, the absorption of the pristine film has a peak centered at $\sim 3.1 \mathrm{eV}$, whereas the DFT calculation gives a peak position at $\sim 4 \mathrm{eV}$. Nevertheless, the structure of the spectra including the number of peaks, their relative positions, and the evolution with the doping ratios are in a good agreement between the calculated and experimental results, both for the glassy and $\beta$-phase, see Figure $2 \mathrm{a}, \mathrm{b}$ and Figure $2 \mathrm{c}, \mathrm{d}$. This agreement leads us to the following assignment of the observed peaks.

As already mentioned in the result part, peak A corresponds to the pristine systems and originates from HOMO-LUMO transitions. Peak D is present for relatively low doping ratios and mainly originates from the excitation from the highest occupied levels in the valence band to the lowest unoccupied levels in the conduction band (transitions marked " $\mathrm{T}_{\mathrm{V}}$ " in Figures 4 and 5). The new assignment is well-supported by UPS results. The onset of the observed peak $\mathrm{H}_{\mathrm{d}}$ in the valence band is $\sim 1.6 \mathrm{eV}$ larger than the onset of the gap state $\mathrm{G}$, corresponding to the energy difference between " $\mathrm{T}_{\mathrm{V}}$ " and " $\mathrm{T}_{\mathrm{P}}$ ". Such values qualitatively match the energy difference between the cutoff of the two absorption peaks, i.e., D and C. This is different from previous consensus assigning the peak $\mathrm{D}$ to the excitation from the valence band to the polaronic state. ${ }^{43}$ As the doping ratio increases to $2 / 3$ electrons per monomer, this peak is no longer present in the spectra. Due to the uneven distribution of $\mathrm{K}$ atoms from the film surface to the substrate, each of the experimental spectra shown in Figure 2a,b is a result of the accumulation of several doping ratios. That is why the peak $\mathrm{D}$ can still be seen in the spectra with doping ratios higher than $50 \%$.

Peaks $B$ and $C$ are assigned to the excitations from the occupied polaronic levels to the lowest unoccupied levels in the conduction band (transitions marked " $\mathrm{T}_{\mathrm{P}}$ " in Figures 4 and 5). It can be noted that the DFT calculations do not reproduce a systematic shift of the peaks $\mathrm{B}$ and $\mathrm{C}$ toward lower energies with the increase in the doping ratio observed in the experiment. Nevertheless, there is some qualitative correspondence between the experiment and simulations because DFT calculations predict typically two or more overlapping peaks at high doping ratios (see Figure $2 \mathrm{c}, \mathrm{d}$ ). It is not clear whether this discrepancy is due to the general limitations of DFT as discussed above or due to factors not accounted for in the calculations. For example, our present DFT calculations do not explicitly include the presence of dopants. The Coulomb interactions between the polarons and the dopants can affect the electronic structure and therefore the peak positions especially at high doping ratios. We plan to study this aspect in more details in the future investigations.
However, we can try to give a reasonable explanation through the experimental data. According to the previous report, only one NIR absorption located at $\sim 0.5 \mathrm{eV}$ has been observed at low doping ratios in solution, and some new features with higher energy $(E \approx 0.5-1 \mathrm{eV})$ emerge at high doping ratios. ${ }^{43}$ By contrast, we find that the only difference in the film is the additionally generated peak B at low doping ratios. Because the distance between the polarons and $\mathrm{K}^{+}$ions in the film is much shorter than that in solution at low doping ratios, the electrostatic interaction should be considered in the film. This interaction changes the polaron orbitals and lowers their energy, which is not taken into account in the DFT calculations. As a result, the excitation energy from polaronic states to the lowest conduction band becomes larger, and a new, blueshifted peak emerges. This explanation is supported by the calculations of p-doped P3HT films that the polaron transforms from "free" to "bound" by shortening the distance between counterions and polymer chains, resulting in a blueshifted mid-IR absorption spectrum. ${ }^{7}$ From the XPS spectra shown in Figure S2, we can see a shoulder peak in the $\mathrm{C} 1 \mathrm{~s}$ core level spectra upon doping. Since the new feature locates at the low binding energy side of the alkyl carbon, it cannot be caused by oxygen, and we attribute it to the $\mathrm{K}^{+}$ interactions. For high doping ratios, $\mathrm{K}^{+}$ions show a weakened effect on polarons due to the screening of the cations deposited before, resulting in a decrease of peak B and a redshift of B and C. However, based on the DFT results, the low-energy peaks of $T_{P}$ at high doping ratios also contribute to the feature $B$. As a result, feature $B$ can be observed all the time in the film.

\section{CONCLUSION}

In summary, we investigate the electronic structures of polarons in K-doped glassy and $\beta$-phase PFO films by UVvis-NIR absorption and photoelectron spectroscopy. DFTbased calculations are used to describe the evolution of absorption peaks in doped polymers with increasing doping ratios. In contrast to the reduction in solution, both films exhibit two NIR absorption peaks (B and C) originating from the excitation from polaronic states to the conduction band. The peak B with higher energy $(\sim 1.0 \mathrm{eV})$ dominating at low doping ratios is attributed to the influence of $\mathrm{K}$ cations. The other peak D located at the visible region is assigned to the excitation from the destabilized valence band to the conduction band, where the destabilized valence band is predicted as a miniband at low doping ratios by DFT calculations. Our computations have described the presence of unpaired electrons and polaron pairs during the whole reduction process from $Q=1$ to 12 ( $\left.c_{\text {red }}=0-200 \%\right)$. Comparing the spectra of the two conformations, we find that their polaron features are almost identical, which is consistent with the calculation results. We believe that our studies can help to better understand the formation of polarons in $\mathrm{PFO}$ films.

\section{ASSOCIATED CONTENT}

\section{Supporting Information}

The Supporting Information is available free of charge at https://pubs.acs.org/doi/10.1021/acs.jpcc.0c08442.

XPS spectra of K-doped glassy and $\beta$-phase films; absorption spectra of doped PFO after exposure to air; $\mathrm{K}$ distribution explored by ARXPS and annealing; calculated orbitals, band diagrams, and absorption 
spectra of amorphous and crystalline $\mathrm{F}_{6}$; spin of electrons and multiplicity of each doping ratio (PDF)

\section{AUTHOR INFORMATION}

\section{Corresponding Author}

Yongzhen Chen - Laboratory of Organic Electronics, Department of Science and Technology, Linköping University, 60174 Norrköping, Sweden; (1) orcid.org/0000-0002-23724007; Email: yongzhen.chen@liu.se

\section{Authors}

Sarbani Ghosh - Laboratory of Organic Electronics, Department of Science and Technology, Linköping University, 60174 Norrköping, Sweden; (1) orcid.org/0000-0002-3012$910 \mathrm{X}$

Xianjie Liu - Laboratory of Organic Electronics, Department of Science and Technology, Linköping University, 60174 Norrköping, Sweden; orcid.org/0000-0002-3190-2774

Igor V. Zozoulenko - Laboratory of Organic Electronics, Department of Science and Technology, Linköping University, 60174 Norrköping, Sweden

Mats Fahlman - Laboratory of Organic Electronics, Department of Science and Technology, Linköping University, 60174 Norrköping, Sweden

Slawomir Braun - Laboratory of Organic Electronics, Department of Science and Technology, Linköping University, 60174 Norrköping, Sweden

Complete contact information is available at:

https://pubs.acs.org/10.1021/acs.jpcc.0c08442

\section{Author Contributions}

${ }^{\dagger}$ Y.C. and S.G. contributed equally to this manuscript.

\section{Notes}

The authors declare no competing financial interest.

\section{ACKNOWLEDGMENTS}

This work was financed by the Knut and Alice Wallenberg Foundation project "Tail of the Sun", the Swedish Foundation for Strategic Research SE13-0060, by the Swedish Research Council (project grant nos. 2016-05498 and 2016-05990), and by the Swedish Government Strategic Research Area in Materials Science on Functional Materials at Linköping University (Faculty Grant SFO Mat LiU no. 2009 00971). The computations were performed on resources provided by the Swedish National Infrastructure for Computing (SNIC) at NSC and HPC2N.

\section{REFERENCES}

(1) Bredas, J. L.; Street, G. B. Polarons, Bipolarons, and Solitons in Conducting Polymers. Acc. Chem. Res. 1985, 18, 309-315.

(2) Fahlman, M.; Fabiano, S.; Gueskine, V.; Simon, D.; Berggren, M.; Crispin, X. Interfaces in Organic Electronics. Nat. Rev. Mater. 2019, 4, 627-650.

(3) Lu, N.; Li, L.; Geng, D.; Liu, M. A Review for Polaron Dependent Charge Transport in Organic Semiconductor. Org. Electron. 2018, 61, 223-234.

(4) Grancini, G.; Maiuri, M.; Fazzi, D.; Petrozza, A.; Egelhaaf, H.-J.; Brida, D.; Cerullo, G.; Lanzani, G. Hot Exciton Dissociation in Polymer Solar Cells. Nat. Mater. 2013, 12, 29-33.

(5) Chew, A. R.; Ghosh, R.; Pakhnyuk, V.; Onorato, J.; Davidson, E. C.; Segalman, R. A.; Luscombe, C. K.; Spano, F. C.; Salleo, A. Unraveling the Effect of Conformational and Electronic Disorder in the Charge Transport Processes of Semiconducting Polymers. Adv. Funct. Mater. 2018, 28, 1804142.

(6) Kahmann, S.; Loi, M. A.; Brabec, C. J. Delocalisation Softens Polaron Electronic Transitions and Vibrational Modes in Conjugated Polymers. J. Mater. Chem. C 2018, 6, 6008-6013.

(7) Ghosh, R.; Luscombe, C. K.; Hambsch, M.; Mannsfeld, S. C. B.; Salleo, A.; Spano, F. C. Anisotropic Polaron Delocalization in Conjugated Homopolymers and Donor-Acceptor Copolymers. Chem. Mater. 2019, 31, 7033-7045.

(8) Peeks, M. D.; Tait, C. E.; Neuhaus, P.; Fischer, G. M.; Hoffmann, M.; Haver, R.; Cnossen, A.; Harmer, J. R.; Timmel, C. R.; Anderson, H. L. Electronic Delocalization in the Radical Cations of Porphyrin Oligomer Molecular Wires. J. Am. Chem. Soc. 2017, 139, 10461-10471.

(9) Salzmann, I.; Heimel, G.; Oehzelt, M.; Winkler, S.; Koch, N. Molecular Electrical Doping of Organic Semiconductors: Fundamental Mechanisms and Emerging Dopant Design Rules. Acc. Chem. Res. 2016, 49, 370-378.

(10) Jacobs, I. E.; Moulé, A. J. Controlling Molecular Doping in Organic Semiconductors. Adv. Mater. 2017, 29, 1703063.

(11) Kiefer, D.; Kroon, R.; Hofmann, A. I.; Sun, H.; Liu, X.; Giovannitti, A.; Stegerer, D.; Cano, A.; Hynynen, J.; Yu, L.; et al. Double Doping of Conjugated Polymers with Monomer Molecular Dopants. Nat. Mater. 2019, 18, 149-155.

(12) Pei, Q.; Yu, G.; Zhang, C.; Yang, Y.; Heeger, A. J. Polymer Light-Emitting Electrochemical Cells. Science 1995, 269, 1086-1088.

(13) Youssef, K.; Li, Y.; O’Keeffe, S.; Li, L.; Pei, Q. Fundamentals of Materials Selection for Light-Emitting Electrochemical Cells. Adv. Funct. Mater. 2020, 30, 1909102.

(14) Flagg, L. Q.; Giridharagopal, R.; Guo, J.; Ginger, D. S. AnionDependent Doping and Charge Transport in Organic Electrochemical Transistors. Chem. Mater. 2018, 30, 5380-5389.

(15) Rivnay, J.; Inal, S.; Salleo, A.; Owens, R. M.; Berggren, M.; Malliaras, G. G. Organic Electrochemical Transistors. Nat. Rev. Mater. 2018, 3, 17086.

(16) Crispin, X.; Jakobsson, F. L. E.; Crispin, A.; Grim, P. C. M.; Andersson, P.; Volodin, A.; van Haesendonck, C.; Van der Auweraer, M.; Salaneck, W. R.; Berggren, M. The Origin of the High Conductivity of Poly(3,4-Ethylenedioxythiophene)-Poly(Styrenesulfonate) (PEDOT-PSS) Plastic Electrodes. Chem. Mater. 2006, 18, 4354-4360.

(17) Bubnova, O.; Khan, Z. U.; Wang, H.; Braun, S.; Evans, D. R.; Fabretto, M.; Hojati-Talemi, P.; Dagnelund, D.; Arlin, J.-B.; Geerts, Y. H.; et al. Semi-Metallic Polymers. Nat. Mater. 2014, 13, 190-194.

(18) Brédas, J. L.; Chance, R. R.; Silbey, R. Comparative Theoretical Study of the Doping of Conjugated Polymers: Polarons in Polyacetylene and Polyparaphenylene. Phys. Rev. B 1982, 26, 58435854.

(19) Campbell, D. K.; Bishop, A. R.; Fesser, K. Polarons in QuasiOne-Dimensional Systems. Phys. Rev. B 1982, 26, 6862-6874.

(20) Baeuerle, P.; Segelbacher, U.; Maier, A.; Mehring, M. Electronic Structure of Mono- and Dimeric Cation Radicals in End-Capped Oligothiophenes. J. Am. Chem. Soc. 1993, 115, 10217-10223.

(21) Österbacka, R.; An, C. P.; Jiang, X. M.; Vardeny, Z. V. TwoDimensional Electronic Excitations in Self-Assembled Conjugated Polymer Nanocrystals. Science 2000, 287, 839-842.

(22) Beljonne, D.; Cornil, J.; Sirringhaus, H.; Brown, P. J.; Shkunov, M.; Friend, R. H.; Brédas, J.-L. Optical Signature of Delocalized Polarons in Conjugated Polymers. Adv. Funct. Mater. 2001, 11, 229234.

(23) Fratiloiu, S.; Grozema, F. C.; Koizumi, Y.; Seki, S.; Saeki, A.; Tagawa, S.; Dudek, S. P.; Siebbeles, L. D. A. Electronic Structure and Optical Properties of Charged Oligofluorenes Studied by VIS/NIR Spectroscopy and Time-Dependent Density Functional Theory. J. Phys Chem. B 2006, 110, 5984-5993.

(24) Guo, J.; Ohkita, H.; Benten, H.; Ito, S. Near-IR Femtosecond Transient Absorption Spectroscopy of Ultrafast Polaron and Triplet Exciton Formation in Polythiophene Films with Different Regioregularities. J. Am. Chem. Soc. 2009, 131, 16869-16880. 
(25) Png, R.-Q.; Ang, M. C. Y.; Teo, M.-H.; Choo, K.-K.; Tang, C. G.; Belaineh, D.; Chua, L.-L.; Ho, P. K. H. Madelung and Hubbard Interactions in Polaron Band Model of Doped Organic Semiconductors. Nat. Commun. 2016, 7, 11948.

(26) Lögdlund, M.; Lazzaroni, R.; Stafström, S.; Salaneck, W. R.; Brédas, J.-L. Direct Observation of Charge-Induced $\pi$-Electronic Structural Changes in a Conjugated Polymer. Phys. Rev. Lett. 1989, 63, 1841-1844.

(27) Braun, S.; Salaneck, W. R. Fermi Level Pinning at Interfaces with Tetrafluorotetracyanoquinodimethane (F4-TCNQ): The Role of Integer Charge Transfer States. Chem. Phys. Lett. 2007, 438, 259-262.

(28) Precht, R.; Stolz, S.; Mankel, E.; Mayer, T.; Jaegermann, W.; Hausbrand, R. Investigation of Sodium Insertion into Tetracyanoquinodimethane (TCNQ): Results for a TCNQ Thin Film Obtained by a Surface Science Approach. Phys. Chem. Chem. Phys. 2016, 18, 3056-3064.

(29) Salaneck, W. R.; Friend, R. H.; Brédas, J. L. Electronic Structure of Conjugated Polymers: Consequences of Electron-Lattice Coupling. Phys. Rep. 1999, 319, 231-251.

(30) Brédas, J. L.; Wudl, F.; Heeger, A. J. Polarons and Bipolarons in Doped Polythiophene: A Theoretical Investigation. Solid State Commun. 1987, 63, 577-580.

(31) Alkan, F.; Salzner, U. Theoretical Investigation of Excited States of Oligothiophene Anions. J. Phys. Chem. A 2008, 112, 6053-6058.

(32) Ghosh, S.; Gueskine, V.; Berggren, M.; Zozoulenko, I. V. Electronic Structures and Optical Absorption of N-Type Conducting Polymers at Different Doping Levels. J. Phys. Chem. C 2019, 123, 15467-15476.

(33) Zozoulenko, I.; Singh, A.; Singh, S. K.; Gueskine, V.; Crispin, X.; Berggren, M. Polarons, Bipolarons, and Absorption Spectroscopy of PEDOT. ACS Appl. Poly. Mater. 2018, 1, 83-94.

(34) Pingel, P.; Neher, D. Comprehensive Picture of P-Type Doping of $\mathrm{P} 3 \mathrm{HT}$ with the Molecular Acceptor $\mathrm{F}_{4}$ TCNQ. Phys. Rev. B 2013, $87,115209$.

(35) Gao, J.; Stein, B. W.; Thomas, A. K.; Garcia, J. A.; Yang, J.; Kirk, M. L.; Grey, J. K. Enhanced Charge Transfer Doping Efficiency in JAggregate Poly(3-Hexylthiophene) Nanofibers. J. Phys. Chem. C 2015, 119, 16396-16402.

(36) Shi, X.; Nádaždy, V.; Perevedentsev, A.; Frost, J. M.; Wang, X.; von Hauff, E.; MacKenzie, R. C. I.; Nelson, J. Relating Chain Conformation to the Density of States and Charge Transport in Conjugated Polymers: The Role of the $\beta$-Phase in Poly $(9,9-$ Dioctylfluorene). Phys. Rev. X 2019, 9, No. 021038.

(37) Bright, D. W.; Dias, F. B.; Galbrecht, F.; Scherf, U.; Monkman, A. P. The Influence of Alkyl-Chain Length on Beta-Phase Formation in Polyfluorenes. Adv. Funct. Mater. 2009, 19, 67-73.

(38) Volkov, A. V.; Singh, S. K.; Stavrinidou, E.; Gabrielsson, R.; Franco-Gonzalez, J. F.; Cruce, A.; Chen, W. M.; Simon, D. T.; Berggren, M.; Zozoulenko, I. V. Spectroelectrochemistry and Nature of Charge Carriers in Self-Doped Conducting Polymer. Adv. Electron. Mater. 2017, 3, 1700096.

(39) Sahalianov, I.; Hynynen, J.; Barlow, S.; Marder, S. R.; Müller, C.; Zozoulenko, I. UV-to-IR Absorption of Molecularly p-Doped Polythiophenes with Alkyl and Oligoether Side Chains: Experiment and Interpretation Based on Density Functional Theory. J. Phys. Chem. B 2020, 124, 11280-11293.

(40) Frisch, M. J.; Trucks, G. W.; Schlegel, H. B.; Scuseria, G. E.; Robb, M. A.; Cheeseman, J. R.; Scalmani, G.; Barone, V.; Petersson, G. A.; Nakatsuji, H.; et al. Gaussian 16 Rev. B.01. Wallingford, CT, 2016.

(41) Krishnan, R.; Binkley, J. S.; Seeger, R.; Pople, J. A. SelfConsistent Molecular Orbital Methods. XX. A Basis Set for Correlated Wave Functions. J. Chem. Phys. 1980, 72, 650-654.

(42) Lin, Y.-S.; Li, G.-D.; Mao, S.-P.; Chai, J.-D. Long-Range Corrected Hybrid Density Functionals with Improved Dispersion Corrections. J. Chem. Theory Comput. 2013, 9, 263-272.

(43) Takeda, N.; Asaoka, S.; Miller, J. R. Nature and Energies of Electrons and Holes in a Conjugated Polymer, Polyfluorene. J. Am. Chem. Soc. 2006, 128, 16073-16082.
(44) Zaikowski, L.; Kaur, P.; Gelfond, C.; Selvaggio, E.; Asaoka, S.; Wu, Q.; Chen, H.-C.; Takeda, N.; Cook, A. R.; Yang, A.; et al. Polarons, Bipolarons, and Side-by-Side Polarons in Reduction of Oligofluorenes. J. Am. Chem. Soc. 2012, 134, 10852-10863.

(45) Cheetham, N. J.; Ortiz, M.; Perevedentsev, A.; Dion-Bertrand, L.-I.; Greetham, G. M.; Sazanovich, I. V.; Towrie, M.; Parker, A. W.; Nelson, J.; Silva, C.; et al. The Importance of Microstructure in Determining Polaron Generation Yield in Poly(9,9-Dioctylfluorene). Chem. Mater. 2019, 31, 6787-6797.

(46) Montilla, F.; Ruseckas, A.; Samuel, I. D. W. Absorption CrossSections of Hole Polarons in Glassy and $\beta$-Phase Polyfluorene. Chem. Phys. Lett. 2013, 585, 133-137.

(47) Liao, L. S.; Cheng, L. F.; Fung, M. K.; Lee, C. S.; Lee, S. T.; Inbasekaran, M.; Woo, E. P.; Wu, W. W. Interface Formation between Poly(9,9-Dioctylfluorene) and Ca Electrode Investigated Using Photoelectron Spectroscopy. Chem. Phys. Lett. 2000, 325, 405-410.

(48) Greczynski, G.; Fahlman, M.; Salaneck, W. R. An Experimental Study of Poly(9,9-Dioctyl-Fluorene) and Its Interfaces with $\mathrm{Li}, \mathrm{Al}$, and LiF. J. Chem. Phys. 2000, 113, 2407-2412.

(49) Fung, M. K.; Lai, S. L.; Bao, S. N.; Lee, C. S.; Lee, S. T.; Wu, W. W.; Inbasekaran, M.; O’Brien, J. J. Interface between Poly (9,9Dioctylfluorene) and Alkali Metals: Cesium, Potassium, Sodium, and Lithium. J. Vac. Sci. Technol. A 2002, 20, 911-918.

(50) Fahlman, M.; Beljonne, D.; Lögdlund, M.; Friend, R. H.; Holmes, A. B.; Brédas, J. L.; Salaneck, W. R. Experimental and Theoretical Studies of the Electronic Structure of Na-Doped Poly (Para-Phenylenevinylene). Chem. Phys. Lett. 1993, 214, 327-332. 